\title{
Nanoscale
}

A) Check for updates

Cite this: Nanoscale, 2022, 14, 3537

\section{Ferroelectric bismuth-titanate nanoplatelets and nanowires with a new crystal structure $\dagger$}

\author{
Darko Makovec, (DD *a,b Nina Križaj, a,b Anton Meden, ${ }^{c}$ Goran Dražić, (ID d \\ Hana Uršič, (iD e Rok Kostanjšek, (iD f Martin Šala (iD d and Sašo Gyergyek (iD) a
}

Received 17th January 2022,

Accepted 11th February 2022

DOI: $10.1039 / \mathrm{d} 2 \mathrm{nr} 00307 \mathrm{~d}$

rsc.li/nanoscale
Two different morphologies of ferroelectric bismuth titanate $\left(\mathrm{Bi}_{4} \mathrm{Ti}_{3} \mathrm{O}_{12}\right)$ nanoparticles, i.e., nanoplatelets and nanowires, were synthesized by changing the concentration of $\mathrm{NaOH}$ during a hydrothermal treatment of precipitated $\mathrm{Ti}^{4+}$ and $\mathrm{Bi}^{3+}$ ions. The nanoparticles' crystal structures were characterized using atomic-resolution imaging with a $C_{S}$-probe-corrected scanning-transmission electron microscope in combination with X-ray diffractometry and Raman spectroscopy. The nanoplatelets $(10 \mathrm{~nm}$ thick and from $50 \mathrm{~nm}$ to $200 \mathrm{~nm}$ wide) exhibit the Aurivillius-type layered-perovskite crystal structure that is characteristic of $\mathrm{Bi}_{4} \mathrm{Ti}_{3} \mathrm{O}_{12}$, whereas the nanowires (from $15 \mathrm{~nm}$ to $35 \mathrm{~nm}$ wide and from several hundreds of $n m$ to several $\mu m$ long) exhibit an entirely new structure with an orthorhombic unit cell ( $a=3.804(1) \AA$, $b=11.816(3) \AA$, and $c=9.704(1) \AA$ ). The nanowire structure is composed of two structural layers alternating along the orthorhombic $c$-direction: a structural layer composed of two parallel layers of $\mathrm{Bi}$ atoms that resembles the $\left(\mathrm{Bi}_{2} \mathrm{O}_{2}\right)^{2+}$ layer of the Aurivillius structure, and a structural layer composed of two parallel layers of $\mathrm{Ti}$ atoms, where every sixth $\mathrm{Ti}$ is replaced with $\mathrm{Bi}$. Observations of the ferroelectric domains with transmission electron and piezo-response force microscopy indicated the ferroelectric nature of both nanostructures. The nanowire structure is a metastable polymorph of the bismuth titanate stabilized at the nanoscale. With annealing at temperatures above $500{ }^{\circ} \mathrm{C}$ the nanowire structure topotactically transforms into the Aurivillius structure.

\section{Introduction}

Bismuth titanate $\left(\mathrm{Bi}_{4} \mathrm{Ti}_{3} \mathrm{O}_{12}, \mathrm{BIT}\right)$ is the most studied and exploited member of a large family of ferroelectric Aurivillius mixed oxides that are characterized by a layered structure composed of bismuth-oxide layers alternating with perovskite-like layers. The BIT structure can therefore be described in terms of two layers: a $\left(\mathrm{Bi}_{2} \mathrm{O}_{2}\right)^{2+}$ layer and a perovskite $\left(\mathrm{Bi}_{2} \mathrm{Ti}_{3} \mathrm{O}_{10}\right)^{2-}$

\footnotetext{
${ }^{a}$ Department for Materials Synthesis, Jožef Stefan Institute, Jamova 39, SI-1000 Ljubljana, Slovenia.E-mail: darko.makovec@ijs.si

${ }^{b} J o z ̌ e f$ Stefan International Postgraduate School, Jamova 39, SI-1000 Ljubljana, Slovenia

${ }^{c}$ Faculty of Chemistry and Chemical Technology, University of Ljubljana, Večna pot 113, SI-1000 Ljubljana, Slovenia

${ }^{d}$ National Institute of Chemistry, Hajdrihova 19, SI-1000 Ljubljana, Slovenia

${ }^{e}$ Department for Electronic Ceramics, Jožef Stefan Institute, Jamova 39, 1000 Ljubljana, Slovenia

${ }^{f}$ Department of Biology, Biotechnical Faculty, University of Ljubljana, Jamnikarjeva 101, SI-1000 Ljubljana, Slovenia

$\dagger$ Electronic supplementary information (ESI) available: List of materials used and additional information on the characterization methods, LeBail profile fitting of the XRD pattern, Raman spectroscopy, TEM/XRD of annealed nanowires, TEM observation of domain structure, and PFM observation of switching and inducing ferroelectric domains. See DOI: 10.1039/d2nr00307d
}

layer stacked along the pseudo-tetragonal $c$-axis. Below the Curie temperature $\left(T_{\mathrm{C}} \sim 675^{\circ} \mathrm{C}\right)$ BIT adopts the monoclinic P1a1 unit cell. ${ }^{1,2}$ The ferroelectric BIT exhibits a modest spontaneous polarization with the larger component of $\sim 50 \mu \mathrm{C}$ $\mathrm{cm}^{-2}$ oriented along the $a$-direction and the smaller component of $\sim 4 \mu \mathrm{C} \mathrm{cm}^{-2}$ along the $c$-direction, a high coercive field, and an excellent fatigue resistance. As a bulk material, BIT is promising for ferroelectric random-access-memory devices and lead-free, high-temperature piezoelectric and pyroelectric devices. ${ }^{3,4}$ Also, in its nano form, BIT is becoming increasingly important for different applications, including nanogenerators for biomechanical energy harvesting, ${ }^{5}$ sensing, ${ }^{6,7}$ visible-light photocatalysis, ${ }^{8-10}$ electrocatalysis, ${ }^{7}$ and piezocatalysis. ${ }^{11,12}$

BIT nanoparticles can be synthesized using a simple hydrothermal treatment of precipitated $\mathrm{Bi}^{3+}$ and $\mathrm{Ti}^{4+}$ ions in an aqueous alkali hydroxide at moderate concentrations. ${ }^{6-9,11-17}$ Because of their layered structure the bismuth titanate nanoparticles tend to grow predominantly in the $a / b$ plane to form 2-D rectangular platelet crystals, i.e., nanoplatelets and nanosheets, with the large surfaces parallel to the (001) crystal planes. ${ }^{7,8,13,14,16,17}$ However, numerous other nanostructures including 1-D nanocrystals (e.g., nanowires, nanobelts, nano- 
bundles, nanorods), ${ }^{7,8,15}$ and 3-D nanostructures assembled from 1-D or 2-D nanoparticles,,$^{6,9,11,12,15,16}$ were synthesized using the hydrothermal method. The morphology of the BIT nanoparticles can be controlled by selecting the synthesis conditions, e.g., source of titania, type of mineralizer, reactant concentrations, reaction temperature and time, and use of surfactants. $^{7,8,15,17}$

In this investigation we synthesized BIT nanoplatelets (NPLs) and nanowires (NWs) just by changing the concentration of the $\mathrm{NaOH}$ during the hydrothermal treatment of precipitated $\mathrm{Bi}^{3+}$ and $\mathrm{Ti}^{4+}$ ions. Their characterization showed that both nanostructures are ferroelectric, but they differ in their crystal structure: the NPLs exhibit the Aurivillius-type structure characteristic for BIT, whereas the NWs exhibit an entirely new layered structure, not described before.

\section{Experimental}

Bismuth-titanate nanoparticles were synthesized with a hydrothermal treatment of separately precipitated $\mathrm{Ti}^{4+}$ and $\mathrm{Bi}^{3+}$ ions. In a typical experiment, $1.0 \mathrm{~g}$ of titanium butoxide was dissolved in $20 \mathrm{~mL}$ of tert-butanol. The $\mathrm{Ti}^{4+}$ ions were precipitated with the addition of $40 \mathrm{~mL}$ of water under vigorous stirring. The white precipitate was separated with centrifugation and washed two times with water and finally with $\mathrm{NaOH}$ at the same concentration as used for the subsequent hydrothermal treatment $\left(0.5 \mathrm{~mol} \mathrm{~L}^{-1}\right.$ or $\left.2 \mathrm{~mol} \mathrm{~L}^{-1}\right)$. In parallel, a stoichiometric amount of $\mathrm{Bi}\left(\mathrm{NO}_{3}\right)_{3}(\mathrm{Bi} / \mathrm{Ti}=4 / 3)$ was dissolved in diluted $\mathrm{HNO}_{3}(0.68 \mathrm{wt} \%)$. The $\mathrm{Bi}^{3+}$ ions were precipitated with the addition of aqueous ammonia (0.94 wt\%) under vigorous stirring to set the final $\mathrm{pH}$ to $\sim 8$. The white precipitate was separated with centrifugation and washed two times with water and finally with $\mathrm{NaOH}$. After washing with $\mathrm{NaOH}$, the colour of the precipitate turned yellow. Finally, both precipitates were quantitatively transferred to a $70 \mathrm{~mL}$ Teflon-lined autoclave and filled with a $\mathrm{NaOH}$ aqueous solution $(0.5 \mathrm{~mol}$ $\mathrm{L}^{-1}$ or $2 \mathrm{~mol} \mathrm{~L}^{-1}$ ) to a final volume of $45 \mathrm{~mL}$. The autoclave was closed and heated for 38 hours at $200{ }^{\circ} \mathrm{C}$. The white product was thoroughly washed with water and dried at $60{ }^{\circ} \mathrm{C}$. The materials used are listed in the ESI. $\dagger$

The BIT nanostructures were characterized with a transmission electron microscope (TEM; Jeol 2010F) and a $C_{\mathrm{S}^{-}}$ probe-corrected scanning-transmission electron microscope (STEM; Jeol ARM 200CF). X-ray powder diffraction (XRD) patterns were recorded with a PANalytical X'Pert Pro MPD diffractometer. The Raman spectra of the samples were recorded with a NT-MDT model Integra Spectra for Materials Science equipped with a confocal microscope. The chemical compositions of the samples were measured with inductively coupled plasma (ICP-OES) using a Varian 715-ES ICP optical emission spectrometer after lithium metaborate fusion digestion. The topography and piezo-response force microscopy (PFM) images were recorded using an atomic force microscope (AFM; Jupiter XR, Asylum Research, CA). Details of the characterization methods are given in the ESI. $\dagger$

\section{Results}

\section{Morpho-structural properties}

The hydrothermal treatment of the mixture of $\mathrm{Bi}$ and $\mathrm{Ti}$ precipitates resulted in the formation of BIT in the form of NPLS at the higher $\mathrm{NaOH}$ concentration of $2 \mathrm{~mol} \mathrm{~L}^{-1}$, whereas the NWs grew at the lower $\mathrm{NaOH}$ concentration of $0.5 \mathrm{~mol} \mathrm{~L}^{-1}$, as evident from the TEM images in Fig. 1(a) and (b). The NPLs were approximately $10 \mathrm{~nm}$ thick and from $50 \mathrm{~nm}$ to $200 \mathrm{~nm}$ wide, whereas the NWs were from $15 \mathrm{~nm}$ to $35 \mathrm{~nm}$ wide and from several hundreds of $\mathrm{nm}$ to several $\mu \mathrm{m}$ long. Quantification of the energy-dispersive X-ray spectra (EDXS) taken from the NPLs and the NWs in the TEM showed similar compositions, with the $\mathrm{Bi} / \mathrm{Ti}$ atomic ratio being $1.3 \pm 0.1$ and $1.2 \pm 0.1$ for the NPLs and the NWs, respectively, matching the composition of the $\mathrm{Bi}_{4} \mathrm{Ti}_{3} \mathrm{O}_{12}$. Sodium, which can be incorporated into the nanoparticles due to the synthesis in $\mathrm{NaOH}$, was not detected.

The compositions of the two morphologies were also confirmed with ICP-OES. The $\mathrm{Bi} / \mathrm{Ti}$ atomic ratio was close to the theoretical value for $\mathrm{Bi}_{4} \mathrm{Ti}_{3} \mathrm{O}_{12}$ of 1.33. The measurement showed $\mathrm{Bi} / \mathrm{Ti}=1.34$ for the NPLs and $\mathrm{Bi} / \mathrm{Ti}=1.40$ for the NWs. The concentration of $\mathrm{Na}$ in the samples with NPLs and NWs was low, i.e., $0.4 \mathrm{wt} \%$ and $0.1 \mathrm{wt} \%$, respectively. Here, it should be noted that we cannot exclude the presence of a small quantity of secondary phases in the analysed samples.

The XRD pattern of the NPLs (Fig. 1(c)) showed broad peaks that match with the Aurivillius (AU) $\mathrm{Bi}_{4} \mathrm{Ti}_{3} \mathrm{O}_{12}$ phase. However, the XRD of the nanowires is completely different. A comprehensive search showed no match with the XRD data for any known phase, suggesting a new crystal structure. A similar

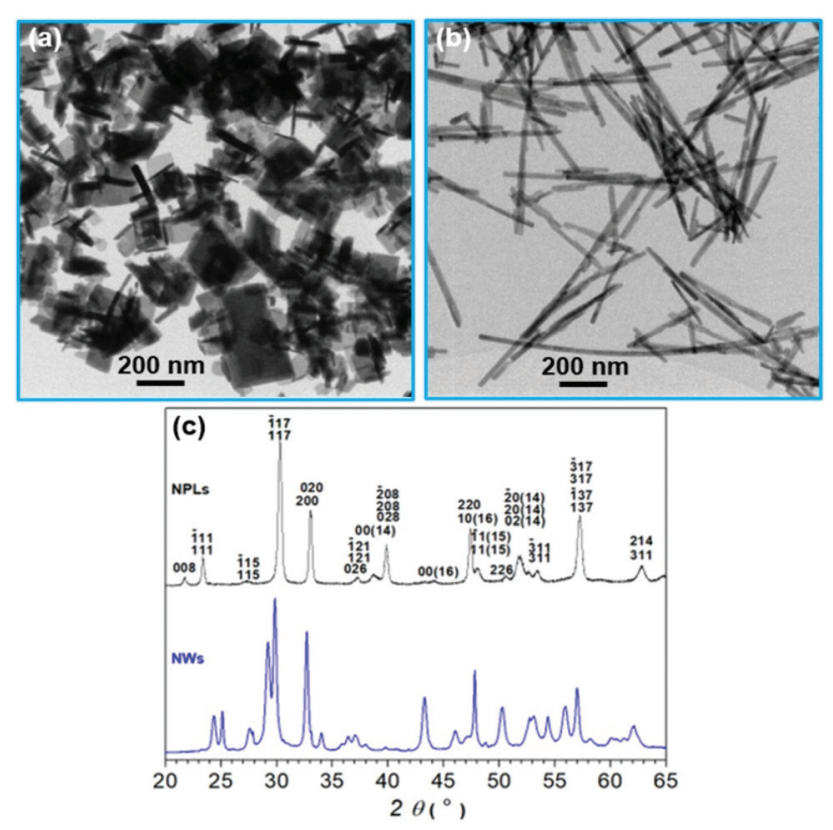

Fig. 1 TEM images ( $a$ and b) and XRD patterns (c) of NPLs (a) and NWs (b). 
XRD pattern was previously reported for BIT nanowires, which the authors ascribed to a mixture of $\mathrm{Bi}_{2} \mathrm{O}_{3}, \mathrm{TiO}_{2}$, and $\mathrm{K}_{2} \mathrm{Ti}_{6} \mathrm{O}_{13} \cdot{ }^{17}$ However, electron diffraction and atomic-resolution imaging with a STEM (discussed below) showed that the material contains just one phase, having an orthorhombic unit cell with the approximate dimensions $a \sim 3.8 \AA, b \sim$ 12.0 ̊, $c \sim 9.5 \AA$. Based on this information it was possible to find an unit cell that gave a satisfactory LeBail fit to the observed powder pattern (see Fig. SI 1 in the ESI $\dagger$ ). ${ }^{18}$ The refined unit-cell parameters were $a=3.804(1) \AA$, $b=11.816(3)$ $\AA$, and $c=9.704(1) \AA$.

The Raman spectroscopy also revealed significant differences in the structure of the two morphologies of bismuth titanate. The bands of internal vibrational modes of the $\mathrm{TiO}_{6}$ octahedron occur for the NWs at significantly higher frequencies than for the NPLs (Fig. SI $2 \dagger$ ). The difference in the position of bands is most likely related to the difference in the $\mathrm{TiO}_{6}$ octahedra's connectivity, which is corner sharing in the NPLs and edge sharing in the NWs (see the ESI $\dagger$ for details). ${ }^{19,20}$

\section{Atomic-resolution STEM imaging}

Differences in the crystal structures of the two bismuth-titanate morphologies were revealed using direct atomic-resolution STEM imaging. Special care was taken during the STEM imaging to avoid excessive degradation of the nanostructures under the electron beam (see the ESI $\dagger$ for details). However, evaporation of the bismuth could not be entirely avoided. The STEM images clearly show the main characteristics of the two different structures. Fig. 2(a) and (b) show STEM images of the NPLs oriented along the $[001]_{\mathrm{AU}}$ direction and the $[110]_{\mathrm{AU}}$ direction, respectively.

The atomic-resolution high-angle annular dark-field (HAADF) STEM images of the NPLs clearly correspond to the layered, Aurivillius-type (AU) structure of $\mathrm{Bi}_{4} \mathrm{Ti}_{3} \mathrm{O}_{12}$. Since the intensity of the individual atomic column in the " $Z$-contrast" HAADF images depends on the column's average atomic number $Z\left(\sim Z^{\alpha} \text { with } \alpha \text { slightly lower than } 2\right)^{21}$ the positions of the various columns in the BIT AU structure can be identified by their brightness. Generally, the columns containing the heavier $\mathrm{Bi}^{3+}$ ions $(Z=83)$ appear much brighter than the columns containing only the $\mathrm{Ti}^{4+}$ ions $(Z=22)$, while the $\mathrm{O}^{2-}$ $(Z=6)$ columns are too light to be detected in the HAADF images. Along the $[001]_{\mathrm{AU}}$ orientation the brightness of the spots in the HAADF image (Fig. 2(a)) remains constant throughout the image because all the cationic columns have the same composition $(4 \mathrm{Bi}+3 \mathrm{Ti})$. When the NPL is oriented along the $[110]_{\mathrm{AU}}$ direction, we can clearly distinguish the columns that contain the $\mathrm{Bi}$ atoms from the much weaker $\mathrm{Ti}$ columns (Fig. 2(b)). The two alternating structural layers, i.e., a $\left(\mathrm{Bi}_{2} \mathrm{O}_{2}\right)^{2+}$ layer and a $\left(\mathrm{Bi}_{2} \mathrm{Ti}_{3} \mathrm{O}_{10}\right)^{2-}$ perovskite-like layer, can be clearly resolved (Fig. 2(b)). Interestingly, the NPLs always terminate at the large (001) surfaces with the $\left(\mathrm{Bi}_{2} \mathrm{O}_{2}\right)^{2+}$ structural layer. The nanoparticles with layered structures, such as the Aurivillius-type structure, normally adopt a specific structure defined by a termination at surfaces with a specific, low-energy atomic layer. $^{22}$
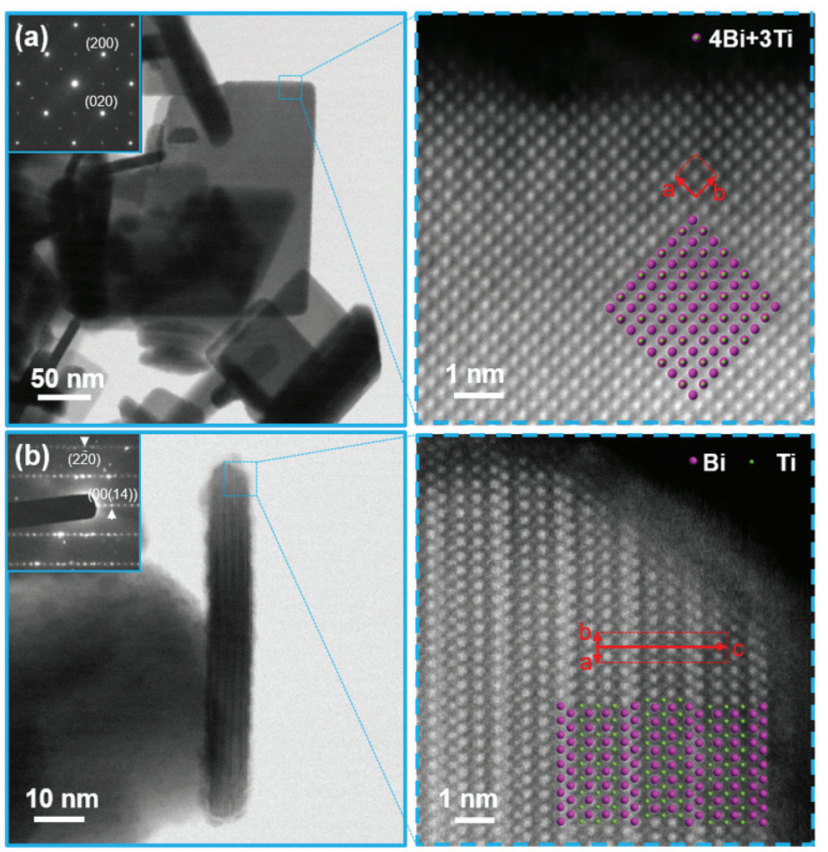

Fig. 2 BF STEM images (insets: corresponding electron-diffraction patterns) and HAADF STEM images of bismuth-titanate NPLs oriented along $[001]_{A U}$ direction (a) and $[110]_{A U}$ direction (b). The projected structural models are superimposed over the images to illustrate the positions of the $\mathrm{Bi}^{3+}$ and $\mathrm{Ti}^{4+}$ ions. The unit cell of the BIT Aurivillius structure is marked with red rectangles on the HAADF images. ${ }^{1}$

The structure of the NWs is very different to that of the NPLs. An analysis of the electron-diffraction patterns suggested an orthorhombic unit cell. Along the $[001]_{\mathrm{NW}}$ direction, the electron-diffraction pattern (Fig. 3(b)) consists of stronger reflections that match with the pattern of the Aurivillius structure in the $[001]_{\mathrm{AU}}$ zone axis (see inset of Fig. 2(a)) and the weaker, superstructural reflections in the direction perpendicular to the NW. The $a$-direction of the new structure was set along the length of the NW with the $a$-parameter of the unit cell being $\sim 3.8 \AA$ A. Interestingly, the superstructural reflections are not periodic, as evident from the corresponding intensity profile in Fig. 3(c). They consist of groups composed of the primary reflections (marked with red arrows in Fig. 3(c)) with a set of weaker satellite reflections characteristic of a multiple diffraction (marked with lines of different colours). The superstructural reflections originate from the structural modulation clearly visible in the corresponding HAADF image (Fig. 3(d)). In the thinner part, near the rim of the NW, every sixth row of spots along the $[010]_{\mathrm{NW}}$ direction is much stronger (marked on Fig. 3(d) with red arrows), suggesting a larger proportion of $\mathrm{Bi}$ atoms. The $b$-parameter of the unit cell was measured to be $\sim 12 \AA$. In the thicker part of the NW, usually two rows have a stronger intensity (marked with blue arrows), or the stronger intensity is distributed over three rows (marked with green arrows). This uneven contrast is a consequence of the stacking faults, i.e., antiphase boundaries, lying perpendicular to the electron beam (visible in the HAADF images taken along the $[100]_{\mathrm{NW}}$ direction, see below). 

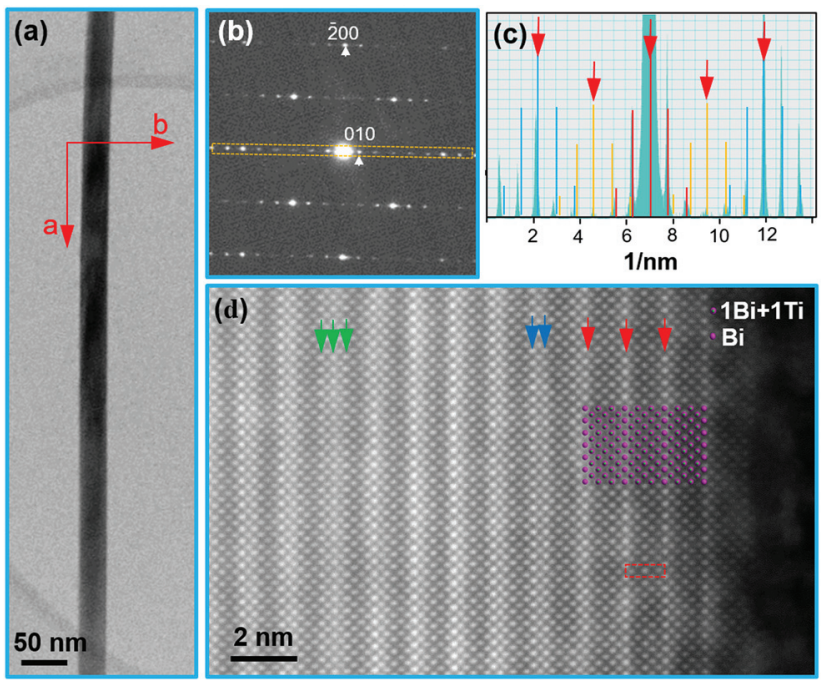

Fig. 3 TEM image (a), electron-diffraction pattern (b) and HAADF STEM image (d) of a bismuth-titanate NW oriented along [001] $]_{N W}$ direction. Fig. 3(c) shows an intensity profile across the row of reflections marked on (c) with a yellow rectangle. The projected model proposed for the NW structure is superimposed over the image (d) to illustrate the positions of the $\mathrm{Bi}^{3+}$ and $\mathrm{Ti}^{4+}$ ions. The unit cell of the NW structure is marked with a red rectangle on the HAADF image (d).

The HAADF image of the NW oriented along the $[010]_{\mathrm{NW}}$ direction (Fig. 4(c)) shows a layered structure with two cationic layers alternating along the $c$-direction: a layer composed of two parallel rows of brighter spots (marked in Fig. 4(c) with "B"), which resembles the $\left(\mathrm{Bi}_{2} \mathrm{O}_{2}\right)^{2+}$ layer from the Aurivillius structure (see Fig. 2(b) for the comparison), and a layer of two parallel rows of weaker spots (marked with "T"). One row of the $\mathrm{Ti}$ atoms in the $\mathrm{T}$ layer is brighter than the other, suggesting that it contains a proportion of the $\mathrm{Bi}$ atoms. The c-parameter of the unit cell was found to be $\sim 9.5 \AA$.

For the STEM imaging of the structure along the $[100]_{\mathrm{NW}}$ direction (i.e., along the nanowire) the NWs were incorporated into a polymer and cut with an ultramicrotome (see the ESI $\dagger$ for details). Also in the $[100]_{\mathrm{NW}}$ zone, the two cationic layers alternating along the $c$-direction, i.e., the $\mathrm{B}$ layer and the $\mathrm{T}$ layer, are clearly resolved in the HAADF images (Fig. 5(a) and (b)). At the (001) surfaces the NW structure always terminates at the B layers. In a zig-zag arrangement of the two rows of spots representing the $\mathrm{T}$ layer every sixth weak spot is replaced by a much brighter spot, consistent with the exchange of $\mathrm{Ti}$ atoms for $\mathrm{Bi}$ atoms. As can be expected from the non-periodic weak reflections in the diffraction pattern of the $[001]_{\mathrm{NW}}$ zone axis (Fig. 3(c)), stacking faults are frequently observed in the (010) planes. Due to the random and partial insertion of extra layers of $\mathrm{Ti}^{4+} / \mathrm{Bi}^{3+}$ ions, the periodicity of the brighter spots in the $\mathrm{T}$ layers locally change to 7 , i.e., locally, every seventh weaker spot is exchanged with a brighter spot (indicated by the blue arrows in Fig. 5(b) and Fig. 6).

Based on HAADF STEM images taken along the three axes we propose a tentative arrangement of the cations in the ortho-
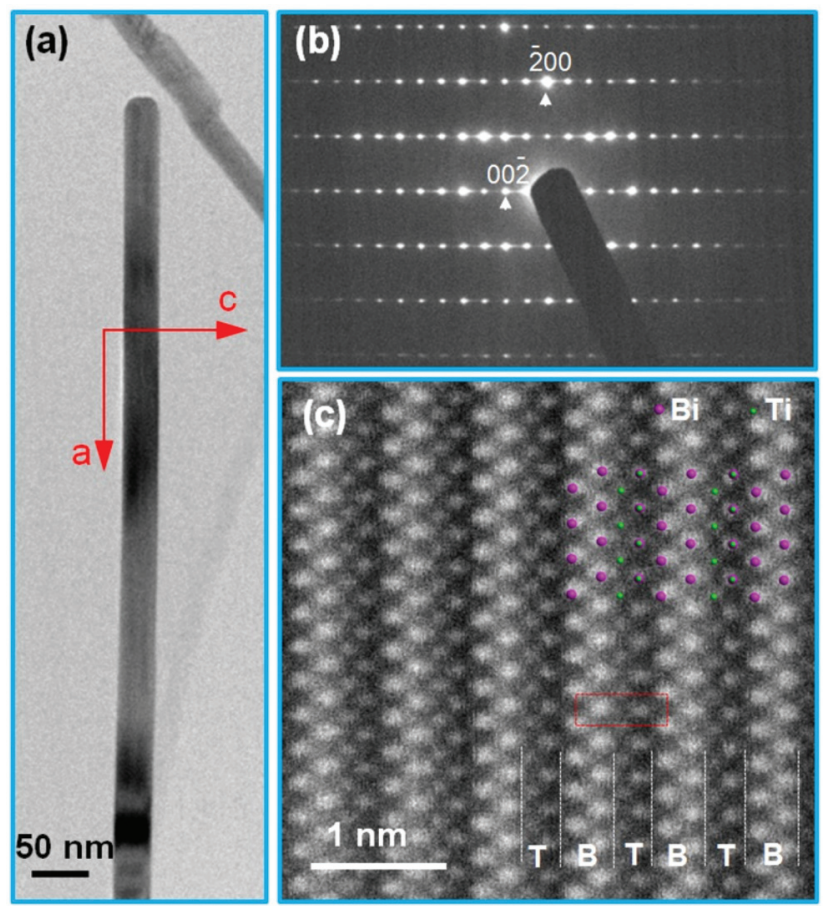

Fig. 4 TEM image (a), electron-diffraction pattern (b) and HAADF STEM image (c) of a bismuth-titanate NW oriented along $[010]_{\mathrm{NW}}$ direction. The projected model proposed for the NW structure is superimposed over the image (c) to illustrate the positions of the $\mathrm{Bi}^{3+}$ and $\mathrm{Ti}^{4+}$ ions. The unit cell of the NW structure is marked with a red rectangle on the HAADF image (c).

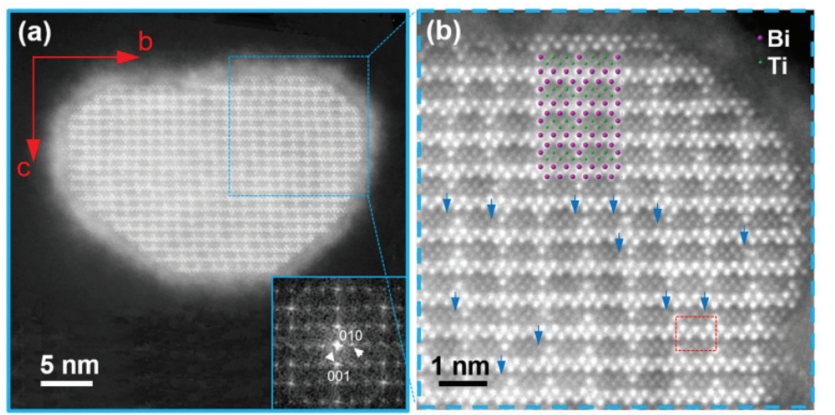

Fig. 5 (a) and (b): HAADF STEM images of a bismuth-titanate NW oriented along $[100]_{\mathrm{NW}}$ direction (inset of (a): a corresponding FFT pattern). The projected model proposed for the NW structure is superimposed over the image (b) to illustrate the positions of the $\mathrm{Bi}^{3+}$ and $\mathrm{Ti}^{4+}$ ions. The unit cell of the NW structure is marked with a red rectangle. Blue arrows indicate some random faults where an extra $\mathrm{Ti}^{4+}$ ion is inserted in the T layer, changing the periodicity of brighter spots from 6 to 7 layers in the $[010]_{\mathrm{NW}}$ direction.

rhombic unit cell of NW structure (Fig. 6). This tentative model of the cation sublattice was superimposed over the HAADF images to illustrate the positions of $\mathrm{Bi}^{3+}$ and $\mathrm{Ti}^{4+}$ ions along all three zone axes (see Fig. 3(e), 4(c) and (b) for [001 $]_{\mathrm{NW}}$, $[010]_{\mathrm{NW}}$, and $[100]_{\mathrm{NW}}$ zone axes, respectively). 


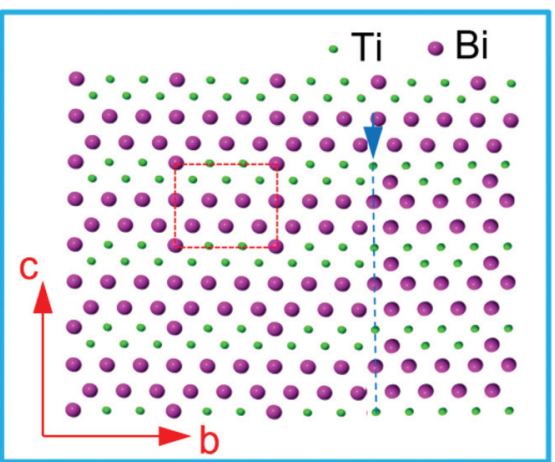

Fig. 6 Cation arrangement in a proposed, tentative model along the $[100]_{\mathrm{NW}}$ direction. Blue dashed line marks a stacking fault where an extra $\mathrm{Ti}^{4+}$ ion (marked with blue arrow) is inserted in a $\mathrm{T}$ layer of the structure.

Topotactic transformation of the nanowire structure to the Aurivillius structure

During annealing at temperatures above $500{ }^{\circ} \mathrm{C}$ the bismuthtitanate NWs transform to the Aurivillius structure. XRD did not show any significant changes after annealing of the NWs for 2 hours at $500{ }^{\circ} \mathrm{C}$, whereas after the annealing for 2 hours at $550{ }^{\circ} \mathrm{C}$ the NWs completely transformed into the AU structure (XRD patterns are given in the ESI, Fig. SI $3 \dagger$ ). After the transformation the particles with the AU structure grow in the form of platelets (Fig. SI $4 \dagger$ ). When the NWs were annealed for 2 hours at $525{ }^{\circ} \mathrm{C}$, they only partially transformed into the $\mathrm{AU}$ structure. Fig. 7 shows HAADF STEM images of a particle from the NW sample annealed at $525{ }^{\circ} \mathrm{C}$. The particle is composed of a NW sandwiched between two lamellas with the AU structure (oriented along $\left([010]_{\mathrm{NW}}||[110]_{\mathrm{AU}}\right.$ zone axes). The coherent interface between the two phases $\left((100)_{\mathrm{NW}}||(110)_{\mathrm{AU}},(001)_{\mathrm{NW}} \|\right.$ $\left.\left.(001)_{\mathrm{AU}}\right),(010) \mathrm{N}_{\mathrm{W}} \|(-110)_{\mathrm{AU}}\right)$ shows a topotactic NW-to-AU transformation. Projected models are superimposed over the atomic-resolution HAADF image of Fig. 5(b) to illustrate the different arrangements of the $\mathrm{Bi}^{3+}$ and $\mathrm{Ti}^{4+}$ cations in the two structures. Note that no precipitation of any secondary phases during the topotactic transformation of the NWs was detected,

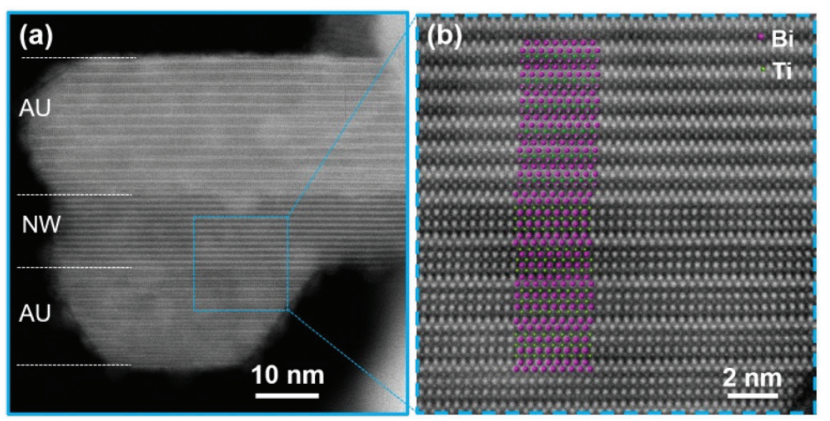

Fig. 7 HAADF STEM images of a BIT particle from the NW sample annealed for 2 hours at $525^{\circ} \mathrm{C}$. The particle is composed of a NW sandwiched between two lamellas with the AU structure $\left([010]_{N w} \|[110]_{A U}\right)$. Projected models of the AU structure and the NW structure are superimposed over Fig. 7(b) to illustrate the positions of the $\mathrm{Bi}^{2+}$ and $\mathrm{Ti}^{4+}$ ions. strongly suggesting that the NWs have the same composition as the $\mathrm{Bi}_{4} \mathrm{Ti}_{3} \mathrm{O}_{12}$ Aurivillius phase.

\section{Ferroelectric properties of the nanoplatelets and the nanowires}

Observation of domain structure. The diffraction patterns taken from the NPLs oriented along $[001]_{\mathrm{AU}}$ (see inset of Fig. 2(a)) show $[010]_{\mathrm{AU}^{-}}$type reflections, which are consistent with a ferroelectric, monoclinic P1a1 phase. ${ }^{2}$ The main component of the polarization for the monoclinic BIT is along the $a$-direction. By following the orientation of the $a$-direction in different parts of an individual NPL $90^{\circ}$-domains were observed (see Fig. SI5 $\dagger$ ).

In the 1-D ferroelectric particles the polarization vector $\boldsymbol{P}$ is usually oriented along their longest dimension. For the ferroelectric NWs the polarization is therefore expected along the $[100]_{\mathrm{NW}}$ direction. The polarity of the crystal structure can be revealed using dark-field (DF) imaging with TEM based on a failure of Friedel's law in the kinematical diffraction. According to Friedel's law a difference in the contrast should be observed when DF images of a non-centrosymmetric structure are formed with $+\boldsymbol{g}$ and $\boldsymbol{- g}$, where the diffraction vector $\boldsymbol{g}$ lies in the direction of $\boldsymbol{P}{ }^{23}$ Consequently, the contrast of a DF image will reverse across a $180^{\circ}$-domain boundary. Fig. 8 shows BF and DF images of a NW. The DF images were formed with (200) and (200) reflections. The contrast in the DF images is alternating along the NW and it is reversed from (200) to (200) for the same area of the NW, in accordance with the existence of $180^{\circ}$-ferroelectric domains, from approximately 50 to

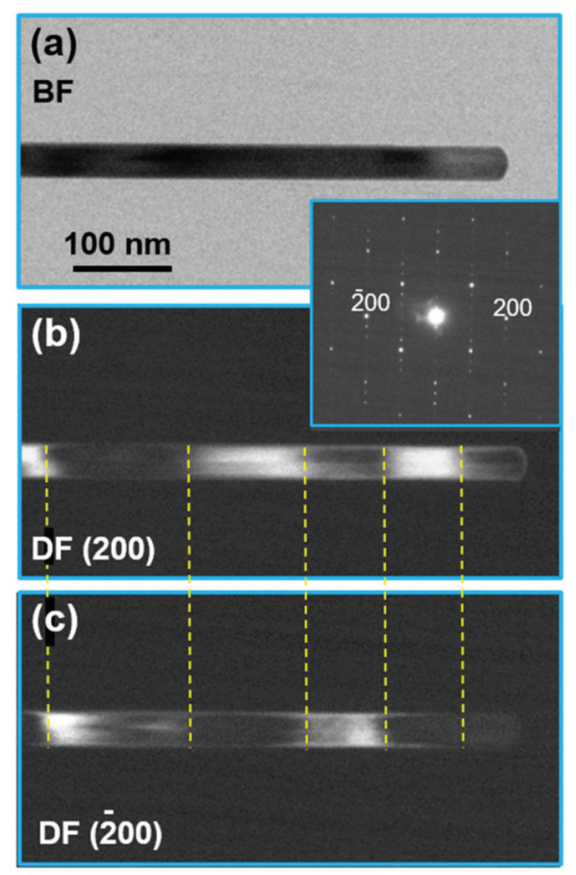

Fig. 8 BF TEM image of a NW oriented along $[001]_{N W}$ direction with corresponding DF images formed with (200) and (200) reflections. Dashed lines mark approximate positions of domain boundaries. 

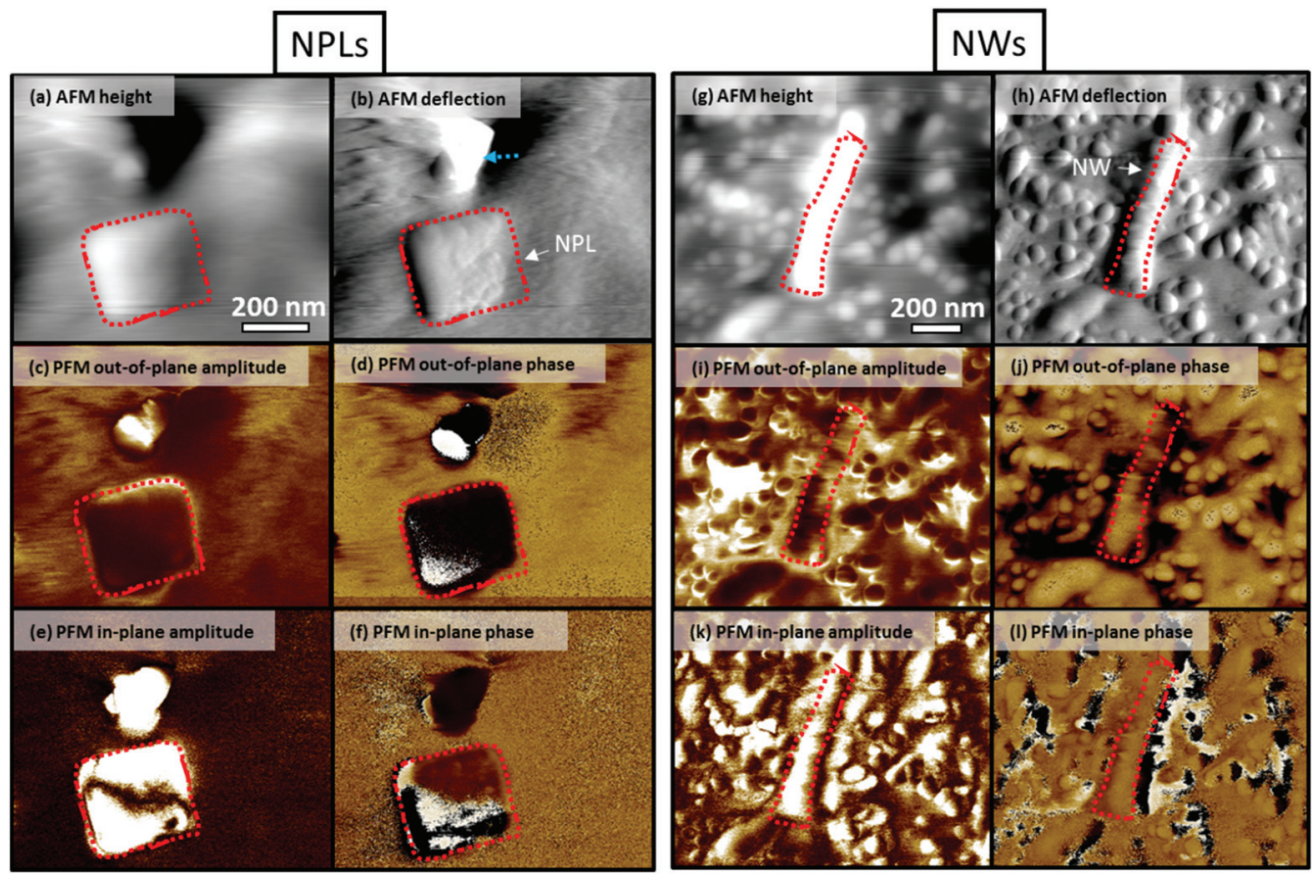

Fig. 9 (a) AFM height and (b) AFM deflection images, (c) PFM out-of-plane amplitude, (d) PFM out-of-plane phase, (e) PFM in-plane amplitude, and (f) PFM in-plane phase images of NPLs. (g) AFM height and (h) AFM deflection images, (i) PFM out-of-plane amplitude, (j) PFM out-of-plane phase, (k) PFM in-plane amplitude, and (l) PFM in-plane phase images of the NWs.

$100 \mathrm{~nm}$ in size. Note that the contrast doesn't change significantly when the DF images are formed with the diffraction vectors $\boldsymbol{g}$ and $-\boldsymbol{g}$ oriented perpendicular to the NW (see Fig. SI6†).

Piezoresponse force microscopy. PFM images of the NPLS inserted into a polymer are shown in Fig. 9(a)-(f). A bundle of overlapping nanoplatelets is marked with a dotted blue arrow in panel (b). The boundary of a single nanoplatelet positioned parallel to the scan plane is marked by a dotted red line. Comparing the AFM height and deflection images (Fig. 9(a) and (b)) with the PFM out-of-plane and in-plane amplitude images of this single plate (Fig. 9(c) and (e)), it is clear that the PFM signal enhancements (bright regions) are observed in the in-plane PFM amplitude signal, indicating a strong polarization in the lateral direction. Moreover, ferroelectric domains are visible in the PFM in-plane amplitude and phase images (Fig. 9(e) and (f)). Similar ferroelectric domains were previously observed in $\mathrm{BaTiO}_{3}$ plates prepared by molten-salt synthesis. ${ }^{24}$ The switching of the ferroelectric domains and the induction of new domains in the in-plane direction could also be observed in the PFM switching spectroscopy experiment, ${ }^{25}$ as shown in the ESI (Fig. SI7†).

PFM images of the NW inserted into the polymer are shown in Fig. 9(g)-(l). The boundary of a single nanowire parallel to the scan plane is marked by a dotted red line. Also in this case, the enhancement of the piezoelectric response is observed in the in-plane PFM amplitude image, indicating a strong polarization towards the longer axis of the nanowire.

\section{Discussion}

The new structure of the NW can be viewed as a metastable polymorph of BIT stabilized at the nanoscale. According to the empirical Ostwald step rule, crystallization occurs in steps in such a way that a thermodynamically unstable phase (i.e., the new nanowire structure) often occurs first because it has a lower nucleation barrier than the stable phase (i.e., the Aurivillius structure). The metastable polymorph remains stable as long as it stays small, if the surface energy of the metastable phase is lower to that of the stable phase. ${ }^{26}$ The polymorphism is well known in simple oxides. Many technologically important nanomaterials, e.g., magnetic maghemite $(\gamma$-Fe2O3) nanoparticles, photocatalytic anatase nanoparticles, $\varepsilon$-Fe2O3 nanoparticles with a giant coercivity, various iron oxide hydroxide pigments, etc., are all metastable phases stabilized at the nanoscale. Recently, we also demonstrated polymorphism in strontium hexaferrite, a technologically important oxide material widely used for magnets exhibiting a complex, layered structure. ${ }^{27}$

During the topotactic transformation of the NWs to the equilibrium Aurivillius structure no secondary phases were precipitated, strongly suggesting that the two phases have the same $\mathrm{Bi}_{4} \mathrm{Ti}_{3} \mathrm{O}_{12}$ composition. Both structures consist of two alternating structural layers. The B layer of the NW structure is composed of two parallel rows of $\mathrm{Bi}$ atoms in a zig-zag arrangement and resembles the $\left(\mathrm{Bi}_{2} \mathrm{O}_{2}\right)^{2+}$ layer of the Aurivillius structure. The T layer of the NW structure is much thinner than the perovskite layer of the Aurivillius structure. It contains only 
two parallel rows of cations, whereas the perovskite layer is composed of 5 rows of cations ( $3 \mathrm{Ti}$ and $2 \mathrm{Bi}$ ). The $\mathrm{T}$ layer can be presented as the two rows of $\mathrm{Ti}$ ions in a zig-zag arrangement, where every sixth Ti is exchanged with Bi (Fig. 5(b) and 6). The arrangement of the $\mathrm{Ti}$ atoms in the $\mathrm{T}$ layer is consistent with two layers of edge-sharing $\left(\mathrm{TiO}_{6}\right)^{2-}$ octahedra, as opposed to the corner-sharing $\left(\mathrm{TiO}_{6}\right)^{2-}$ octahedra of the perovskite layers in the $\mathrm{AU}$ structure, where the $\mathrm{Ti}^{4+}$ ions are stacked one above each other. Such an arrangement of the $\mathrm{Ti}^{4+}$ ions in the two structures is consistent with the results of the Raman spectroscopy.

The proposed cation arrangement in the NW structure predicts a $\mathrm{Bi}$ : Ti ratio of $7: 5$, which is only slightly richer in $\mathrm{Bi}$ (58.33 at\% of cations) than $4: 3$ (57.14 at\% of cations). The excess $\mathrm{Bi}$, predicted by the model, is compensated by a random partial insertion of the Ti layers parallel to the (010) planes, visible along the [100] direction (Fig. 5(b) and explained there).

Both BIT nanostructures, the NPLs and the NWs, are piezoelectric. An enhancement of the piezoelectric signal in the inplane direction is observed for both NPLs and NWs, indicating a strong polarization in the longitudinal direction of the particles. Some tens-of-nanometres ferroelectric domains are clearly visible in the NPLs. Moreover, switching and the inducing of new domains were observed. On the other hand, the domain structure of NWs was not visible in the PFM scans, probably because the resolution of the technique is above 20 nanometres. However, the $180^{\circ}$-domains were clearly visible on the DF TEM images of the NWs (Fig. 8).

\section{Conclusions}

Bismuth titanate $\left(\mathrm{Bi}_{4} \mathrm{Ti}_{3} \mathrm{O}_{12}\right)$ nanowires and nanoplatelets were synthesized with a hydrothermal treatment of the precipitated $\mathrm{Ti}^{4+}$ and $\mathrm{Bi}^{3+}$ ions in aqueous $\mathrm{NaOH}$ with the concentration of $0.5 \mathrm{~mol} \mathrm{~L}^{-1}$ and $2 \mathrm{~mol} \mathrm{~L}^{-1}$, respectively. The nanoplatelets (10 nm thick and from $50 \mathrm{~nm}$ to $200 \mathrm{~nm}$ wide) exhibit the Aurivillius-type layered-perovskite crystal structure characteristic of $\mathrm{Bi}_{4} \mathrm{Ti}_{3} \mathrm{O}_{12}$, whereas the nanowires (from $15 \mathrm{~nm}$ to $35 \mathrm{~nm}$ wide and from several hundreds of $\mathrm{nm}$ to several $\mu \mathrm{m}$ long) exhibit an entirely new structure, not described before. X-ray diffractometry showed an orthorhombic unit cell of the new nanowire structure $(a=3.804(1) \AA, b=11.816(3) \AA$, and $c=$ 9.704(1) A). Atomic-resolution, high-angle annular dark-field imaging with a $C_{\mathrm{S}}$-probe-corrected scanning-transmission electron microscope showed the cation arrangement in two structural layers alternating along the orthorhombic $c$-direction: a structural layer composed of two parallel layers of $\mathrm{Bi}$ atoms that resembles the $\left(\mathrm{Bi}_{2} \mathrm{O}_{2}\right)^{2+}$ layer of the Aurivillius-type structures, and a structural layer composed of two parallel layers of the $\mathrm{Ti}$ atoms, where every sixth $\mathrm{Ti}$ is replaced by Bi. Such an arrangement of cations in the nanowire structure is consistent with a $\mathrm{Bi}$ : Ti ratio of $7: 5$. This discrepancy in the composition is compensated by a random partial insertion of the Ti layers. The basic arrangement of $\mathrm{Ti}$ in the structural layer is consist- ent with two layers of edge-sharing $\left(\mathrm{TiO}_{6}\right)^{2-}$ octahedra, also suggested by the results of the Raman spectroscopy. With annealing at temperatures above $500{ }^{\circ} \mathrm{C}$ the nanowire structure topotactically transforms into the Aurivillius structure without the precipitation of any secondary phases, proving that the nanowire structure is a metastable polymorph of the $\mathrm{Bi}_{4} \mathrm{Ti}_{3} \mathrm{O}_{12}$ stabilized at the nanoscale. The observation of ferroelectric domains with transmission electron microscopy and piezoresponse force microscopy suggested the ferroelectric nature of both bismuth-titanate polymorphs.

\section{Author contributions}

D. M., G. D., A. M. and H. U. designed the experimental work programme, methodology, supervised the research and drafted the manuscript. N. K. synthesized the nanoparticles. S. G. and R. K. prepared TEM samples using ultramicrotome. D. M. carried out electron microscopy, G. D. analysed the STEM results. A. M. conducted XRD structure determination and proposed the tentative structural model. S. G. conducted Raman analysis. H. U. carried out the PFM measurements. M. S. performed ICP-OES analysis. All authors discussed and revised the manuscript.

\section{Conflicts of interest}

There are no conflicts to declare.

\section{Acknowledgements}

The authors acknowledge the financial support from the Slovenian Research Agency (ARRS) for research core funding No. P2-0089, J2-3041, P1-0034, P2-0105, and the support of Infrastructural Centre "Microscopy of Biological Samples" at Biotechnical faculty, University of Ljubljana. The authors thank B. Anželak, V. Fišinger and J. Cilenšek for help in the laboratory.

\section{References}

1 B. Aurivillius, Ark. Kemi, 1949, 1, 499-512.

2 D. Urushihara, M. Komabuchi, N. Ishizawa, M. Iwata, K. Fukuda and T. Asaka, J. Appl. Phys., 2016, 120, 142117.

3 T. Jardiel, A. C. Caballero and M. Villegas, J. Ceram. Soc. Jpn., 2008, 116, 511-518.

4 A. Moure, Appl. Sci., 2018, 8, 62.

5 H. Li, L. Su, S. Kuang, Y. Fan, Y. Wu, Z. L. Wang and G. Zhu, Nano Res., 2017, 10, 785-793.

6 J. Hu, Y. Yu, H. Guo, Z. Chen, A. Li, X. Feng, B. Xi and G. Hu, J. Mater. Chem., 2011, 21, 5352-5359.

7 Z. Chen, Y. Yu, H. Guo and J. Hu, J. Phys. D: Appl. Phys., 2009, 42, 125307. 
8 L. Xue, G. Qing-Feng, L. Hai-Bo, L. Hong-Ji, B. Chun-Hua and D. Hai-De, Acta Phys.-Chim. Sin., 2012, 28, 1481-1488.

9 S. Niu, R. Zhang, X. Zhang, J. Xiang and C. Guo, Ceram. Int., 2020, 46, 6782-6786.

10 T. Xiao, C. Guo, H. Wanga, R. Zhang, Y. Li, W. Shao, Y. Zhang, X. Wu, J. Tan and W. Ye, Mater. Lett., 2020, 269, 127679.

11 S. Tu, H. Huang, T. Zhang and Y. Zhang, Appl. Catal., B, 2017, 219, 550-562.

$12 \mathrm{~J} . \mathrm{Wu}$, N. Qin, E. Lin, B. Yuan, Z. Kang and D. Bao, Nanoscale, 2019, 11, 21128-21136.

13 Q. Yang, Y. Li, Q. Yin, P. Wang and Y.-B. Cheng, J. Eur. Ceram. Soc., 2003, 23, 161-166.

14 H. Gu, Z. Hu, Y. Hu, Y. Yuan, J. You and W. Zou, Colloids Surf., A, 2008, 315, 294-298.

15 F. Wang, J. Wang, X. Zhong, B. Li, J. Liu, D. Wu, D. Mo, D. Guo, S. Yuan, K. Zhang and Y. Zhou, CrystEngComm, 2013, 15, 1397-1403.

16 F. Wang, J. B. Wang, X. L. Zhong, B. Li, Y. Zhang, C. J. Lu, W. N. Ye and Y. C. Zhou, EPL, 2013, 103, 37002.

17 G. Xu, Y. Yang, H. Bai, J. Wang, H. Tian, R. Zhao, X. Wei, X. Yang and G. Han, CrystEngComm, 2016, 18, 2268-2274.
18 S. A. A. Coelho, J. Appl. Crystallogr., 2018, 51, 210-218.

19 S. Kojima, R. Imaizumi, S. Hamazaki and M. Takashige, Jpn. J. Appl. Phys., 1994, 33, 5559-5564.

20 U. Balachandran and N. G. Eror, J. Solid State Chem., 1982, 42, 276-282.

21 A.C.T. Koch, Determination of Core Structure Periodicity and Point Defect Density along Dislocations, Ph.D. Thesis, Arizona State University, 2002.

22 D. Makovec, B. Belec, T. Goršak, D. Lisjak, M. Komelj, G. Dražić and S. Gyergyek, Nanoscale, 2018, 10, 1448014491.

23 R. Serneels, M. Snykers, P. Delavignette, R. Gevers and S. Amelinckx, Phys. Status Solidi B, 1973, 58, 277292.

24 M. Maček Kržmanc, B. Jančar, H. Uršič, M. Tramšek and D. Suvorov, Cryst. Growth Des., 2017, 17, 3210-3220.

25 H. Uršič and U. Prah, Proc. R. Soc. London, Ser. A, 2019, 475, 20180782-1-20180782-25.

26 A. Navrotsky, Proc. Natl. Acad. Sci. U. S. A., 2004, 101, 12096-12101.

27 D. Makovec, G. Dražić, S. Gyergyek and D. Lisjak, CrystEngComm, 2020, 22, 7113-7122. 